•综述・

\title{
物种分布模型在大型真菌红色名录评估及 保护中的应用：以冬虫夏草为例
}

\author{
李熠 1,2 唐志尧 ${ }^{3}$ 间昱晶 ${ }^{3,4}$ 王 科 2,5 蔡 䂞 ${ }^{2}$ \\ 贺金生 ${ }^{3}$ 古 松 ${ }^{6}$ 姚一建 ${ }^{2 *}$ \\ 1 (扬州大学食品科学与工程学院, 扬州, 江苏 225127) \\ 2 (中国科学院微生物研究所真菌学国家重点实验室, 北京 100101) \\ 3 (北京大学城市与环境学院, 北京 100871) \\ 4 (Center for Macroecology, Evolution and Climate, Natural History Museum of Denmark, University of Copenhagen, Denmark) \\ 5 (中国科学院大学, 北京 100049) \\ 6 (南开大学生命科学学院, 天津 300071)
}

\begin{abstract}
摘要：我国大型真菌资源丰富, 由于受气候变化和人类活动等的影响, 近年来很多物种受到不同程度的威胁, 亟 待保护。红色名录评估是物种保护的第一步, 为有效保护我国大型真菌多样性, 2016年生态环境部和中国科学院联 合启动中国大型真菌红色名录评估项目。合理的评估依赖于完善的物种地理分布、种群数量规模及其动态变化信 息。大型真菌评估信息较少, 需要引入新的方法解决评估信息不足的问题。冬虫夏草(Ophiocordyceps sinensis)是 一种重要的食药用菌, 具有较高的经济价值, 受到全世界的广泛关注, 评估信息相对充足, 此次被评为易危物种。 利用物种分布模型对冬虫夏草未来分布区变化的预测在评估过程中发挥了重要作用。为了将物种分布模型分析方 法引入大型真菌的受威胁等级评估, 本文以此前我们利用物种分布模型预测冬虫夏草的潜在分布区及其对气候变 化响应的研究为例, 介绍了应用物种分布模型预测大型真菌的潜在分布区、未来气候变化情景下分布区变化趋势 的方法和流程, 以及在应用中可能存在的问题和解决方案。通过本文的分析, 我们认为物种分布模型在大型真菌 的红色名录评估和保护中具有重要的应用潜力, 值得推广应用。
\end{abstract}

关键词: 物种分布模型; 冬虫夏草; 真菌保护; 生物多样性

\section{Incorporating species distribution model into the red list assessment and conservation of macrofungi: A case study with Ophiocordyceps sinensis}

\author{
$\mathrm{Yi} \mathrm{Li}^{1,2}$, Zhiyao Tang ${ }^{3}$, Yujing $\mathrm{Yan}^{3,4}$, Ke Wang ${ }^{2,5}$, Lei Cai ${ }^{2}$, Jinsheng $\mathrm{He}^{3}$, Song $\mathrm{Gu}^{6}$, Yijian $\mathrm{Yao}^{2 *}$ \\ 1 College of Food Science and Engineering, Yangzhou University, Yangzhou, Jiangsu 225127 \\ 2 State Key Laboratory of Mycology, Institute of Microbiology, Chinese Academy of Sciences, Beijing 100101 \\ 3 College of Urban and Environmental Sciences, Peking University, Beijing 100871 \\ 4 Center for Macroecology, Evolution and Climate, Natural History Museum of Denmark, University of Copenhagen, \\ Denmark \\ 5 University of Chinese Academy of Sciences, Beijing 100049 \\ 6 College of Life Sciences, Nankai University, Tianjin 300071
}

\begin{abstract}
China is rich in macrofungal biodiversity. However, many species have been threatened in recent years by human activity and climate change. Red list assessment is the first step towards species conservation. To protect this group of fungi, the Ministry of Ecology and Environment of the People's Republic of China and the Chinese Academy of Sciences launched the Red List Assessment of Macrofungi in China in 2016. A reasonable assessment largely relies on the sufficient information of species' geographic information, population numbers and sizes and population dynamics, which is lacked in most of macrofungal species. It is
\end{abstract}


therefore necessary to employ new approaches to find and utilize more information for the assessment. Among the assessed species, Ophiocordyceps sinensis, which is an edible and medicinal fungus endemic to the Tibetan Plateau and its surrounding regions, has relatively abundant information. This species gained attention worldwide due to its obvious economic value and its importance to local societies. A species distribution modeling has also been an important component of its red list assessment. Here, we call on a previous study that aimed to predict the current potential distribution and to project the future distribution of Ophiocordyceps sinensis, and then we discuss how this modeling method can be employed in red list assessments to predict the current potential distribution and the range shifts of other macrofungal species in response to climate change. Challenges of using the model and possible solutions are also discussed. The species distribution modeling method is considered to have great potential for red list assessments and the subsequent conservation of macrofungi.

Key words: species distribution models; Ophiocordyceps sinensis; fungal conservation; biodiversity

\section{中国大型真菌红色名录评估概况}

2016年, 原环境保护部和中国科学院联合启动 中国大型真菌红色名录评估项目，2018年5月22日， 《中国生物多样性红色名录一大型真菌卷》官方 发布。该名录首次对我国已知的9,302个大型真菌物 种的受威胁状况进行了评估, 由于缺少评估信息, 6,340 个物种被评为数据不足(DD), 占评估物种总 数的 $68.16 \%$ (姚一建等, 2020)。

冬虫夏草(Ophiocordyceps sinensis)是一种重要 的珍稀食药用菌, 由真菌侵染鳞翅目蝙蝠蛾科部分 昆虫(Wang \& Yao, 2011)的地下幼虫形成, 仅分布 在我国青海、西藏、四川、云南、甘肃5省区, 以及 喜马拉雅山南麓尼泊尔、印度、不丹等国的部分地 区(Li et al, 2011)。冬虫夏草的利用在青藏高原及周 边国家和地区有着悠久的历史, 是产区农牧民重要 的经济收入来源, 在维持青藏高原生态系统的稳定 性方面发挥着重要作用。受全球气候变化和过度采 挖等因素的影响, 冬虫夏草的分布范围和产量近年 来出现萎缩。采挖冬虫夏草带来的社会和生态问题 (Sharma, 2004; Negi et al, 2006; Winkler et al, 2008; Cannon et al, 2009)以及气候变化对冬虫夏草的影响 (Shrestha et al, 2014; Yan et al, 2017; Hopping et al, 2018)等近年来引起了国际社会的广泛关注。鉴于冬 虫夏草的重要性及其在真菌生物资源保护研究中 的代表性, 为更好促进大型真菌整体的保护, 有人 提议将冬虫夏草作为真菌保护生物学研究的旗舰 物种(Cannon, 2011), 我国也有学者提议将其作为 中国的国菌(Zhang et al, 2012)。
冬虫夏草是此次评估中评估信息较为充足的 物种之一, 物种分布模型 (species distribution models, SDMs) 分析在冬虫夏草受威胁等级评估中 发挥了重要作用, 提供了重要的数据信息。为促进 大型真菌红色名录的评估和保护工作, 本文以冬虫 夏草为例, 介绍了应用物种分布模型预测大型真菌 潜在分布区以及未来气候变化情景下分布区变化 趋势的程序和方法, 以及应用中可能存在的问题及 解决方案, 探讨物种分布模型在大型真菌受威胁等 级评估和物种保护中的应用潜力, 为大型真菌红色 名录的评估和保护提供参考。

\section{物种分布模型与冬虫夏草红色名录评估}

物种分布模型又称环境生态位模型 (environmental niche models, ENMs)、生境适宜度模型(habitat suitability models, HSMs)、潜在生境分布模型 (potential habitat distribution models, PHDMs)等(Guisan et al, 2013), 主要依据物种现有的分布信息和 环境变量数据, 运用特定的算法建立二者之间的定 量关系, 估计物种生存所需的环境条件, 包括温 度、降水、土壤、植被等, 进而模拟物种潜在的地 理分布以及全球气候变化情景下物种分布区的变 化等, 是生态学、生物地理学、进化生物学、保护 生物学中常用的研究方法。SDMs分析在冬虫夏草 受威胁等级评估中提供的物种当前分布、气候变化 情景下物种分布区的变化等信息, 是此次评估的重 要依据。

\section{1 建模方法及过程}

物种分布模型分析包括基础分布数据(occu- 
rrence data)的获取、环境变量(environmental variables)的选择和数据处理、模型选择和评价、未来气 候情景(representative concentration pathways, RCPs) 及扩散模式的选择、建模分析等几个阶段。Yan等 (2017)对冬虫夏草物种分布模型分析方法及过程见 框图1，相关代码已在网站https://github.com/saltp awpaw/OS_distribution_modeling/上公布。

\section{2 模型分析结果及其验证}

模型结果包含了冬虫夏草的适生生境、潜在分 布区及未来气候变化情景下冬虫夏草分布区的变 化趋势, 并通过一系列方法对上述结果进行验证。 Yan等(2017)对冬虫夏草物种分布的分析结果及验 证方法见框图2。

\section{3 冬虫夏草受威胁等级评估}

在此次中国大型真菌红色名录评估中, 冬虫夏 草是评估信息相对充足的物种之一。其地理分布明 确, 生物学特性研究较多, 大量研究人员及当地的 农牧民积累了多年的种群动态变化信息。根据模型 预测, 气候变化可能对冬虫夏草种群和分布产生较 大影响(Yan et al, 2017)。除了气候变化, 过度采挖也 是影响冬虫夏草产量和分布的重要因素(Hopping et $\mathrm{al}, 2018)$ 。此外，过度放牧、采挖活动(搭建帐篷、 砍伐灌木、遗留不可降解生活垃圾)、基础设施建设

\section{框图1: Yan等(2017)对冬虫夏草物种分布模型分析方法及过程：}

1. 基础分布数据: Yan等(2017)共使用了分布于中国和尼泊尔境 内的覆盖冬虫夏草不同分布区和生境类型的218条详细分布 记录。

2. 环境变量的选择: Yan等(2017)从29个气候、植被、土壤变量 中去除共线性后选取了 17 个环境变量, 并将所有数据图层空 间分辨率取 $30 \mathrm{~s}$ (近似 $1 \mathrm{~km} \times 1 \mathrm{~km})$ 。

3. 模型分析方法和模型构建：Yan等(2017)利用分类树分析 (classification tree analysis, CTA)、广义加性模型(generalized additive models, GAM)、广义线性模型(generalized linear models, GLM)、多元自适应样条回归(multivariate adaptive regression, MARS)、最大熵模型(maximum entropy, MaxEnt)、随机森林 (random forest, RF) 6种算法构建了 72个模型, 经模型优度比 较后篮选出 1 种 MaxEnt模型和 3 种 RF 模型构建组合模型 (ensemble model, Araújo \& New, 2007)进行最后的建模分析。

4. 未来气候变化情景选择: 根据对不同气候模型在青藏高原地 区模拟效果的评估结果(Su et al, 2013)，Yan等(2017)选择5个 大气环流模型(general circulation models, GCMs)及其平均值 对未来气候变化情景下冬虫夏草分布区的变化进行预测。未 来气候变化情景取RCP2.6、RCP8.5两种极端碳排放假设, 预 测的时间节点为2050年及2070年。
框图2: Yan等(2017)对冬虫夏草物种分布模型分析结果及其验证:

1. 模型分析结果表明最暖季降水(PWAQ)、最暖季均温 (MTWQ)、最湿季降水(PWEQ)和草本植物覆盖度 $(\mathrm{HV})$ 是影响 冬虫夏草分布的主要环境因素, 适生生境类型包括高山草甸 和亚高山灌从, 适生地土壤类型为薄层土和冻土; 冬虫夏草 当前潜在分布区包括我国青海、西藏、四川、云南、甘肃5 省区以及喜马拉雅山脉南麓尼泊尔、印度、不丹以及缅甸的 部分地区; 在零扩散情景(non-dispersal scenario)下, 冬虫夏 草分布区在未来三、五十年内将萎缩 $36 \%-39 \%$, 青藏高原边 缘及低海拔地区将不再适合冬虫夏草生长。

2. 经检验, 模型预测的环境变量与分布点气象站实测数据接 近; 模型预测出的潜在分布区与此前实地调查和文献记录一 致(Li et al, 2011); 模型预测出的各个国家和中国五省区的分 布比例与目前了解到的各产区的产量基本一致; 冬虫夏草分 布的海拔范围以及各海拔梯度所占的比例也与实地调查结果 接近。从以上 4 个角度可以说明模型分析的可靠性。

等人为因素也在不同程度上导致了冬虫夏草分布 地生境的退化。根据IUCN红色名录评估标准中的A 类标准，如果物种的种群在过去或未来有所减少， 不管这种减少的趋势是来源于观测还是估计、推断 或猜测的结果, 减少的幅度 $\geq 80 \%$ 即可评为极危, $\geq$ $50 \%$ 可评为濒危, $\geq 30 \%$ 评为易危。在我们多年来对 冬虫夏草产地的实地调查过程中，不同产区的采集 者均反映冬虫夏草单位面积的产量明显减少。通过 模型预测，冬虫夏草的分布区受气候变化的影响在 未来三、五十年内可能萎缩36\%-39\% (Yan et al, 2017), 而这一影响是不可逆的。如果考虑所有可能 的影响因素，冬虫夏草分布区和种群的萎缩可能接 近或超过 $50 \%$ (对应濒危等级)。鉴于冬虫夏草相对 于同类真菌来说分布面积较广, 且生物量相对较大, 综合各因素考虑, 我们将其受威胁等级定为易危, 评估主要依据的标准为“A2acd + 3cd” (表1)。

\section{3 模型分析的局限性及解决方㲾}

\section{1 分布数据}

大量研究表明, SDMs分析在预测物种的生态 位、潜在分布区以及未来气候变化情景下分布区的 变化等方面可以提供重要参考信息。但在实际操作 过程中, 模型预测结果的可靠性受分析方法、分布 数据等诸多因素影响(Morán-Ordóñez et al, 2017)。 分布数据是SDMs分析的基础, 建模所用的分布地 点数据越多越准确, 对物种分布范围以及生境类型 的覆盖越全面，模型预测的结果越可靠。而错误的 
分布记录, 尤其是那些落到物种实际分布区外的分 布点信息则可能导致预测结果出现严重偏差(Foody, 2011)。由于相关文献中冬虫夏草分布地点的记录往 往不够精确, 缺少必要的经纬度和海拔信息, 部分 文献中甚至出现由于物种鉴定错误或信息记录错 误等原因导致的不可靠的分布记录(Li et al, 2011), 比如部分海拔低于 $3,000 \mathrm{~m}$ 的分布点(郝剑瑾等, 2009)等, 因此我们在预测时主要采用了本研究组 实地调查记录的分布点信息, 以及少量经过确认的 文献报道的分布点数据。对其他大型真菌来说, 由 于资源调查力度和基础研究的不足, 大量物种的分 布信息还很缺乏(李熠等, 2020), SDMs分析在一定 程度上还需要依赖文献或标本记录。文献记录、馆 藏标本以及其他来源的分布数据中可能存在的错 误一方面可以通过模型分析等方法剔除(Aggarwal, 2013; Liu et al, 2018), 另一方面分类学家也可以参 与对数据质量的把控。利用SDMs分析尽管在一定 程度上可以降低红色名录评估中对物种分布和种 群动态变化数据的依赖, 但目前对绝大多数真菌物 种来说, 已有的分布数据还不足以进行SDMs分析, 未来还需要加强大型真菌的调查和监测研究。对标 本馆馆藏标本的系统整理、鉴定和信息化也是获得 更多物种分布数据的有效途径。

\section{2 模型预测的准确性及结果的合理解释和运用}

除了受基础分布数据的影响, 模型选择、建模 方法等诸多因素也会影响模型预测的可靠性。由于 模型分析未能考虑生物间的相互作用等因素, 模型 预测出的分布范围和面积在大多数情况下比实际 的分布范围更广 (overestimation, Pineda \& Lobo, 2009)。在冬虫夏草的建模分析过程中, 我们得到的 物种当前潜在分布除了文献报道的地点外，还有多 达64个县是无调查记录和文献报道数据支持的, 这 其中除了极个别地点需要进一步调查以外, 其余大 部分特别是远离青藏高原的地点以及海拔低于 3,000 $\mathrm{m}$ 的地区是不大可能有冬虫夏草分布的( Li et al, 2011; Yan et al, 2017)。预测出的冬虫夏草适生区 总面积3.54 $\times 10^{5} \mathrm{~km}^{2}$ 可能远大于实际的分布面积, 模型预测的结果应当审慎采用。尽管物种的分布范 围和分布面积在SDMs分析中可能会或多或少地被 高估, 但模型预测提供的种群和分布区变化幅度等 信息在物种受威胁等级评估中仍可发挥重要作用, 尤其是在大型真菌评估信息缺乏且难以获取的情
况下, SDMs分析提供的分布区和种群变化信息显 得尤为重要。

要获得相对可靠的预测结果, 减少模型的高估, 除了分布数据的准确性和覆盖度外, 模型的选择、 建模分析方法等也很重要。模型的选择是SDMs分 析的核心, 不同的模型可能产生差异极大的结果。 自SDMs分析方法建立以来, 已有多种不同的模型 被开发和应用(许仲林等, 2015), 建模分析过程中对 不同模型的评价是必不可少的，ROC (receiver operating characteristic)和TSS (true skill statistic)值 检测是模型检验的常用方法 (Hanley \& McNeil, 1982; Allouche et al, 2006)。除此之外, 采用机器学 习算法(machine-learning algorithms) 以及组合模型 (ensemble/ consensus model)也是提高预测结果准确 性的有效方式(Marmion et al, 2009; Kindt, 2018)。 Shrestha和Bawa (2014)曾经采用单一模型最大熵模 型对气候变化情景下尼泊尔冬虫夏草产地的变化 进行预测, 其结果与采用组合模型预测的结果(Yan et al, 2017; Hopping et al, 2018)存在显著差异, 前者 的预测结果与实地观测以及采挖人员的长期经验 明显不符, 产生这种差异的原因, 除了所用分布数 据不同外, 模型选择也是重要的影响因素。

物种分布模型对物种适生地及其变化的预测 通常仅考虑环境因子, 而忽略物种相互作用、物种 的迁移扩散能力、物种适应新环境的能力等其他影 响物种分布的重要因素(李国庆等, 2013)。腐生的真 菌受物种相互作用的影响可能小于寄生和共生真 菌, 对温度、水分等环境条件要求较高的物种适应 新环境的能力更弱, 而大型真菌的迁移扩散能力往 往跟孢子的扩散方式相关。对冬虫夏草来说, 尽管 其菌丝体可以在土壤中存活, 但其有性生殖需要寄 主的参与。冬虫夏草的寄主很多都是狭域分布物种 (Wang \& Yao, 2011), 迁移能力有限, 这在很大程度 上会影响物种的扩散和适应新环境的能力。因此, 我们在冬虫夏草的研究中倾向于采用零扩散假设 (non-dispersal scenario), 将预测出的新增分布区视 为无效分布区，预测的丧失面积即为净丧失面积 (Yan et al, 2017)。目前尽管还没有有效方法对物种 的相互作用与物种迁移扩散能力之间的关系进行 研究, 但在应用SDMs预测结果对大型真菌进行受 威胁等级评估时，应根据实际情况选择零扩散、不 受限扩散、有限扩散等不同的假设。 
表1 冬虫夏草评估信息表

Table 1 Assessment information of Ophiocordyceps sinensis

\begin{tabular}{llll}
\hline 分类地位 Taxonomy & & & \\
\hline 界 Kingdom 门 Phylum & 纲 Class & 目 Order & 科 Family \\
真菌界 Fungi $\quad$ 子囊菌门 Ascomycota & 座囊菌纲 Sordariomycetes & 肉座菌目 Hypocreales & 线虫草科 Ophiocordycipitaceae \\
学名 Scientific name & Ophiocordyceps sinensis & \\
中文名 Chinese name & 冬虫夏草 & \\
命名人 Species authority & (Berk.) G.H. Sung, J.M. Sung, Hywel-Jones \& Spatafora \\
分类备注 Taxonomic notes & 三Cordyceps sinensis (Berk.) Sacc. \\
& 三Sphaeria sinensis Berk. \\
& 无性型名称 Hirsutella sinensis X.J. Liu, Y.L. Guo, Y.X. Yu \& W. Zeng \\
& anamorph: Hirsutella sinensis X.J. Liu, Y.L. Guo, Y.X. Yu \& W. Zeng
\end{tabular}

\section{评估信息 Assessment information}

红色名录等级及标准

易危 Vulnerable (VU), A2acd + 3cd

Red list category \& criteria

评估年份 Year published

2016

评估日期 Date assessed

$2016 / 9 / 27$

评定人 Assessor(s)

庄文颖, 李熠 Wen-Ying Zhuang, Yi Li

审定人 Reviewer(s)

吴兴亮, 李春如 Xing-Liang Wu, Chun-Ru Li

描述 Justification

相对于其他虫草类真菌, 冬虫夏草分布范围较广、种群密度和生物量更高, 由于受到人类过度采 挖的影响, 其种群密度明显下降, 气候变化也影响了其分布范围。根据模型预测的结果, 气候变 化导致的冬虫夏草分布区的丧失在未来的三、五十年内可能达到 $30 \%$ 以。

Ophiocordyceps sinensis has a wider distribution, higher population density and biomass comparing with other Cordyceps s. l. species. The population density was observed to decline due to over-harvesting, and its distribution was also reported to be affected by climate change. According to a study with species distribution modeling, over $30 \%$ of its current habitats will be lost in the next 30 to 50 years in response to future climate change.

\section{地理分布 Geographic range}

分布区 Range description

甘肃、青海、四川、云南、西藏 Gansu, Qinghai, Sichuan, Yunnan, Tibet

分布国家 Countries occurrence

中国、尼泊尔、印度、不丹 China, Nepal, India, Bhutan

分布图 Range map

\section{种群 Population}

种群数量 Population size

种群趋势 Current population trend 衰退 Decreasing

附件信息 Additional data

\section{生境 Habitat}

生境 Habitat

高寒草甸、高山灌丛 Alpine meadow, alpine shrub

生态系统 Ecosystems

世代年限 Generation length (years)

\section{商业用途 Use and trade}

商业用途 Use and trade

珍稀食药用菌 A precious edible and medicinal fungus

威胁因子 Threats

主要威胁因子 Major threat (s)

\section{保护行动 Conservation actions}

保护行动 Conservation actions
气候变化、过度采挖 Climate change and over harvesting

该物种1999年被原林业部和农业部列为国家二级保护物种, 其分布地部分被保护区覆盖。建议 对物种的种群动态进行监测, 选择合适的地点建立保护地, 采取必要的保护措施, 尤其是防止 过度采挖利用, 减少采挖活动对其生境的影响。

Ophiocordyceps sinensis has been listed as endangered species under the Chinese Second Class of State Protection by the State Forestry Administration and Ministry of Agriculture since 1999. Part of its distribution areas is now covered by nature reserves. Suggested conservation actions include monitoring the bacterial population dynamics, selecting suitable distribution sites as natural reserves, developing essential protection measures to reduce the influence of collecting activity to its natural habitats, especially to prevent over-harvesting. 


\section{3 其他因素}

SDMs 分析结果仅能反映气候变化对物种的影 响, 人类活动导致的种群退化目前还无法通过 SDMs进行有效评估。对冬虫夏草来说，过度采挖、 过度放牧导致的栖息地质量退化对冬虫夏草的种 群和分布也有一定的影响(Yan et al, 2017; Hopping et al, 2018)。大型真菌的生存状况与栖息地的质量 密切相关, 我国有使用食药用真菌的传统, 很多物 种长期以来被过度采挖, 不科学的采挖方式也影响 了物种的繁殖和种群的稳定。建立监测点, 对重要 物种进行长期监测是了解人类活动对大型真菌影 响的重要途径。

\section{物种分布模型在大型真菌中的应用展望}

冬虫夏草的SDMs分析实践表明, 在确保物种 分布数据的准确性和覆盖度的前提下, 完全可以在 大型真菌中推广使用SDMs, 为大型真菌受威胁等 级评估和物种保护研究提供有价值的信息。尽管 SDMs分析在实际应用中还存在各种问题(Araújo \& Guisan, 2006), 但其在确立物种的生态位、适生地预 测、适生地未来变化趋势等方面有着不可替代的作 用。在动植物中, SDMs被广泛应用于物种潜在分布 区的预测(崔绍朋等, 2018; 李灿等, 2018)、气候变 化情景下物种分布区的变化(Barrows et al, 2010; Liu et al, 2014; Luo et al, 2015)、受威胁物种的保护 和管理(张先锋等, 1994)等。在真菌中, 类似的研究 报道还很少, 目前仅见于冬虫夏草(Shrestha \& Bawa, 2014; Yan et al, 2017; Hopping et al, 2018)和 猪苓(Polyporus umbellatus, 刘蒙蒙等, 2014; Guo et al, 2019)和松口蘑(Tricholoma matsutake, Guo et al, 2017)等少数几个物种。随着国家对大型真菌保护的 重视, 以及SDMs分析方法的不断完善, 相信SDMs 在大型真菌保护中会有越来越广泛的应用, SDMs 分析方法在大型真菌受威胁等级评估、灭绝风险评 估、生物多样性保护、优先保护区确立等方面将会 有更多的研究报道。除此之外, SDMs分析结果结合 物种的多样性分布分析还可以对物种未来的多样 性分布格局进行预测, 为保护区的选址、保护策略 的制定提供参考。SDMs分析结果结合物种种群密 度及其动态变化的实地监测结果还有助于我们了 解大型真菌的资源储量, 实现大型真菌资源的合理 利用。

\section{参考文献}

Aggarwal CC (2013) Outlier Analysis. Springer, New York. Allouche O, Tsoar A, Kadmon R (2006) Assessing the accuracy of species distribution models: Prevalence, kappa and the true skill statistic (TSS). Journal of Applied Ecology, 43, 1223-1232.

Araújo MB, Guisan A (2006) Five (or so) challenges for species distribution modelling. Journal of Biogeography, 33, 1677-1688.

Araújo MB, New M (2007) Ensemble forecasting of species distributions. Trends in Ecology and Evolution, 22, 42-47.

Barrows CW, Rotenberry JT, Allen MF (2010) Assessing sensitivity to climate change and drought variability of a sand dune endemic lizard. Biological Conservation, 143, 731-736.

Cannon PF (2011) The caterpillar fungus, a flagship species for conservation of fungi. Fungal Conservation, 1, 35-39.

Cannon PF, Hywel-Jones NL, Maczey N, Norbu L, Tshitila, Samdup T, Lhendup P (2009) Steps towards sustainable harvest of Ophiocordyceps sinensis in Bhutan. Biodiversity and Conservation, 18, 2263-2281.

Cui SP, Luo X, Li CW, Hu HJ, Jiang ZG (2018) Predicting the potential distribution of white-lipped deer using the MaxEnt model. Biodiversity Science, 26, 171-176. (in Chinese with English abstract) [崔绍朋, 罗晓, 李春旺, 胡慧建, 蒋志刚 (2018) 基于MaxEnt模型预测白唇鹿的潜在分布区. 生物 多样性, 26, 171-176.]

Foody GM (2011) Impacts of imperfect reference data on the apparent accuracy of species presence-absence models and their predictions. Global Ecology and Biogeography, 20, 498-508.

Guisan A, Tingley R, Baumgartner JB, Naujokaitis-Lewis I, Sutcliffe PR, Tulloch AI, Regan TJ, Brotons L, McDonald-Madden E, Mantyka-Pringle C, Martin TG, Rhodes JR, Maggini R, Setterfield SA, Elith J, Schwartz MW, Wintle BA, Broennimann O, Austin M, Ferrier S, Kearney MR, Possingham HP, Buckley YM (2013) Predicting species distributions for conservation decisions. Ecology Letters, 16, 1424-1435.

Guo YL, Li X, Zhao ZF, Wei HY, Gao B, Gu W (2017) Prediction of the potential geographic distribution of the ectomycorrhizal mushroom Tricholoma matsutake under multiple climate change scenarios. Scientific Reports, 7, 46221.

Guo YL, Li X, Zhao ZF, Nawaz Z (2019) Predicting the impacts of climate change, soils and vegetation types on the geographic distribution of Polyporus umbellatus in China. Science of the Total Environment, 648, 1-11.

Hanley JA, McNeil BJ (1982) The meaning and use of the area under a receiver operating characteristic (ROC) curve. 
Radiology, 143, 29-36.

Hao JJ, Cheng Z, Liang HH, Yang XL, Li S, Zhou TS, Zhang WJ, Chen JK (2009) Genetic differentiation and distributing pattern of Cordyceps sinensis in China revealed by rDNA ITS sequences. Chinese Traditional and Herbal Drugs, 40, 112-116. (in Chinese with English abstract) [郝剑瑾, 程舟, 梁洪卉, 杨晓伶, 李珊, 周铜水, 张文驹, 陈家宽 (2009) 基于rDNA ITS序列探讨我国冬虫夏草的遗传分化及分布 格局. 中草药, 40, 112-116.]

Hopping KA, Chignell SM, Lambin EF (2018) The demise of caterpillar fungus in the Himalayan region due to climate change and overharvesting. Proceedings of the National Academy of Sciences, USA, 115, 11489-11494.

Kindt R (2018) Ensemble species distribution modelling with transformed suitability values. Environmental Modelling \& Software, 100, 136-145.

Li C, Liu XA, Wang J, Peng PH, Shao HY, Xian W (2018) Potential distribution and habitat suitability assessment of Taxus chinensis based on MaxEnt in Sichuan Province. Forest Inventory and Planning, 43(1), 22-29. (in Chinese with English abstract) [李灿, 刘贤安, 王娟, 彭培好, 邵怀 勇, 仙巍 (2018) 基于MaxEnt模型的四川省红豆杉潜在 分布区分析及适宜性评价. 林业调查规划, 43(1), 22-29.]

Li GQ, Liu CC, Liu YG, Yang J, Zhang XS, Guo K (2013) Advances in theoretical issues of species distribution models. Acta Ecologica Sinica, 33, 4827-4835. (in Chinese with English abstract) [李国庆, 刘长成, 刘玉国, 杨军, 张 新时, 郭柯 (2013) 物种分布模型理论研究进展. 生态学 报, 33, 4827-4835.]

Li Y, Wang XL, Jiao L, Jiang Y, Li H, Jiang SP, Lhosumtseiring N, Fu SZ, Dong CH, Zhan Y, Yao YJ (2011) A survey of the geographic distribution of Ophiocordyceps sinensis. The Journal of Microbiology, 49, 913-919.

Li Y, Liu DM, Wang K, Wu HJ, Cai L, Cai L, Li JS, Yao YJ (2020) Red list assessment of macrofungi in China: Challenges and measures. Biodiversity Science, 28, 66-73. (in Chinese with English abstract) [李熠, 刘冬梅, 王科, 吴海军, 蔡蕾, 蔡否, 李俊生, 姚一建 (2020) 中国大型 真菌红色名录评估中存在的问题及今后的对策. 生物多 样性, 28, 66-73.]

Liu C, White M, Newell G (2018) Detecting outliers in species distribution data. Journal of Biogeography, 45, 164-176.

Liu MM, Xing YM, Guo SX (2015) Habitat suitability assessment of medicinal Polyporus umbellatus in China based on MaxEnt modeling. China Journal of Chinese Meteria Medica, 40, 2792-2795. (in Chinese with English abstract) [刘蒙蒙, 邢咏梅, 郭顺星 (2015) 基于MaxEnt生态位模 型预测药用真菌猪苓在中国潜在适生区. 中国中药杂志, 40, 2792-2795.]

Liu YP, Yu DY, Xun B, Sun Y, Hao RF (2014) The potential effects of climate change on the distribution and productivity of Cunninghamia lanceolata in China. Environmental Monitoring and Assessment, 186, 135-149.

Luo ZH, Zhou SR, Yu WD, Yu HL, Yang JY, Tian YH, Zhao $\mathrm{M}$, Wu H (2015) Impacts of climate change on the distribution of Sichuan snub-nosed monkeys (Rhinopithecus roxellana) in Shennongjia area, China. American Journal of Primatology, 77, 135-151.

Marmion M, Parviainen M, Luoto M, Heikkinen RK, Thuiller W (2009) Evaluation of consensus methods in predictive species distribution modelling. Diversity and Distributions, 15, 59-69.

Morán-Ordóñez A, Lahoz-Monfort JJ, Elith J, Wintle BA (2017) Evaluating 318 continental-scale species distribution models over a 60-year prediction horizon: What factors influence the reliability of predictions? Global Ecology and Biogeography, 26, 371-384.

Negi CS, Koranga PR, Ghinga HS (2006) Yar tsa Gumba (Cordyceps sinensis): A call for its sustainable exploitation. International Journal of Sustainable Development \& World Ecology, 13, 165-172.

Pineda E, Lobo JM (2009) Assessing the accuracy of species distribution models to predict amphibian species richness patterns. Journal of Animal Ecology, 78, 182-190.

Sharma S (2004) Trade of Cordyceps sinensis from high altitudes of the Indian Himalaya: Conservation and biotechnological priorities. Current Science, 86, 1614-1619.

Shrestha UB, Bawa KS (2014) Impact of climate change on potential distribution of Chinese caterpillar fungus (Ophiocordyceps sinensis) in Nepal Himalaya. PLoS ONE, 9, e106405.

Su F, Duan X, Chen D, Hao Z, Cuo L (2013) Evaluation of the global climate models in the CMIP5 over the Tibetan Plateau. Journal of Climate, 26, 3187-3208.

Wang XL, Yao YJ (2011) Host insect species of Ophiocordyceps sinensis: A review. ZooKeys, 127, 43-59.

Winkler D (2008) Yartsa Gunbu (Cordyceps sinensis) and the fungal commodification of Tibet's rural economy. Economic Botany, 62, 291-306.

Xu ZL, Peng HH, Peng SZ (2015) The development and evaluation of species distribution models. Acta Ecologica Sinica, 35, 557-567. (in Chinese with English abstract) [许 仲林, 彭焕华, 彭守璋 (2015) 物种分布模型的发展及评 价方法. 生态学报, 35, 557-567.]

Yan YJ, Li Y, Wang WJ, He JS, Yang RH, Wu HJ, Wang XL, Jiao L, Tang ZY, Yao YJ (2017) Range shifts in response to climate change of Ophiocordyceps sinensis, a fungus endemic to the Tibetan Plateau. Biological Conservation, 206, 143-150.

Yao YJ, Wei JC, Zhuang WY, Cai L, Liu DM, Li JS, Wei TZ, Li Y, Wang K, Wu HJ (2020) Development of red list assessment of macrofungi in China. Biodiversity Science, 
28, 4-10. (in Chinese with English abstract) [姚一建, 魏江 春, 庄文颖, 蔡蕾, 刘冬梅, 李俊生, 魏铁铮, 李熠, 王 科, 吴海军 (2020) 中国大型真菌红色名录评估研究进 展. 生物多样性, 28, 4-10.]

Zhang XF, Wang D, Wang KX (1994) VORTEX model and its application on the management of Chinese river dolphin (Lipotes vexillifer) population. Chinese Biodiversity, 2, 133-139. (in Chinese with English abstract) [张先锋, 王丁,
王克雄 (1994) 游涡模型及其在白暨豚种群管理中的应 用. 生物多样性, 2, 133-139.]

Zhang YJ, Li EW, Wang CS, Li YL, Liu XZ (2012) Ophiocordyceps sinensis, the flagship fungus of China: Terminology, life strategy and ecology. Mycology, 3, 2-10.

(责任编委：郭良栋 责任编辑：黄祥忠) 